\title{
Monolithic Spiropyran-Based Porous Polysilsesquioxanes with Stimuli-Responsive Properties
}

Daniel Euchler ${ }^{l}$, Caroline R. Ehgartner ${ }^{1}$, Nicola Hüsing ${ }^{1}$, Andrea Feinle ${ }^{1,2 *}$

${ }^{1}$ Chemistry and Physics of Materials, Paris-Lodron University Salzburg, Jakob-Haringer Straße 2a, 5020 Salzburg, Austria.

${ }^{2}$ Chemistry and Chemical Biology, McMaster University, 1280 Main Street West, Hamilton, ON, L8S 4M1, Canada.

*Corresponding author: andrea.feinle@sbg.ac.at
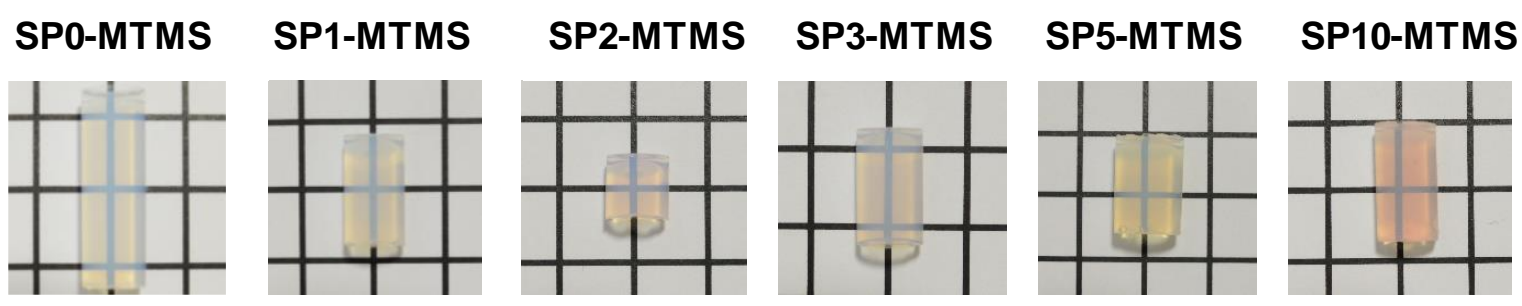

$\downarrow$ UV
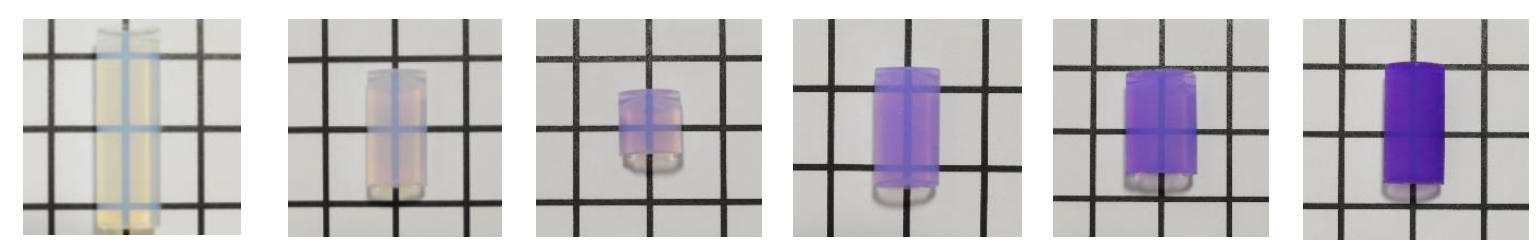

Figure S1. Prepared monoliths before and after UV light irradiation. 

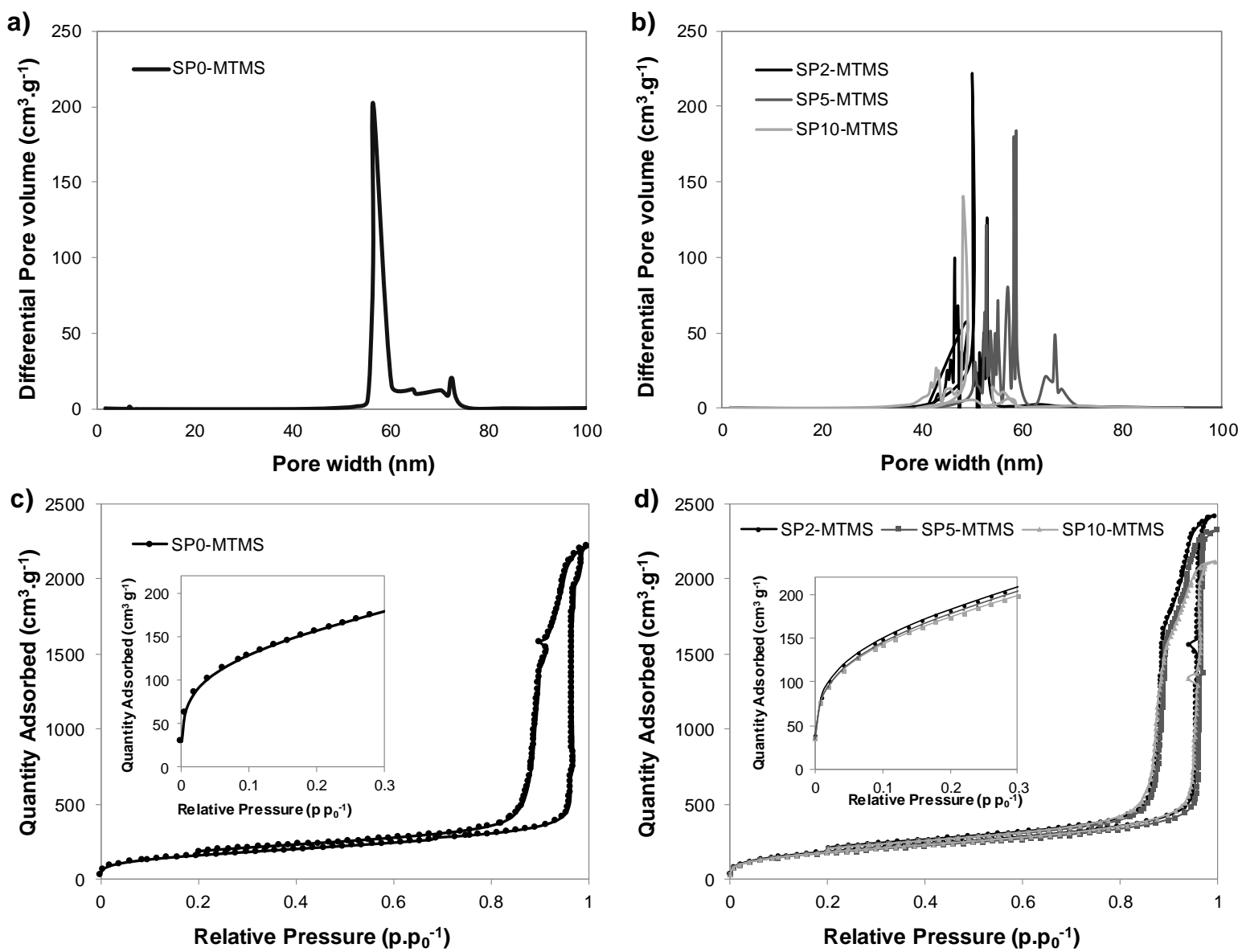

Figure S2. Pore size distribution $(a, b)$ and the related adsorption-desorption isotherms (c,d) obtained by nitrogen sorption measurements for the samples SP0-MTMS, SP2-MTMS, SP5-MTMS, and SP10-MTMS. 

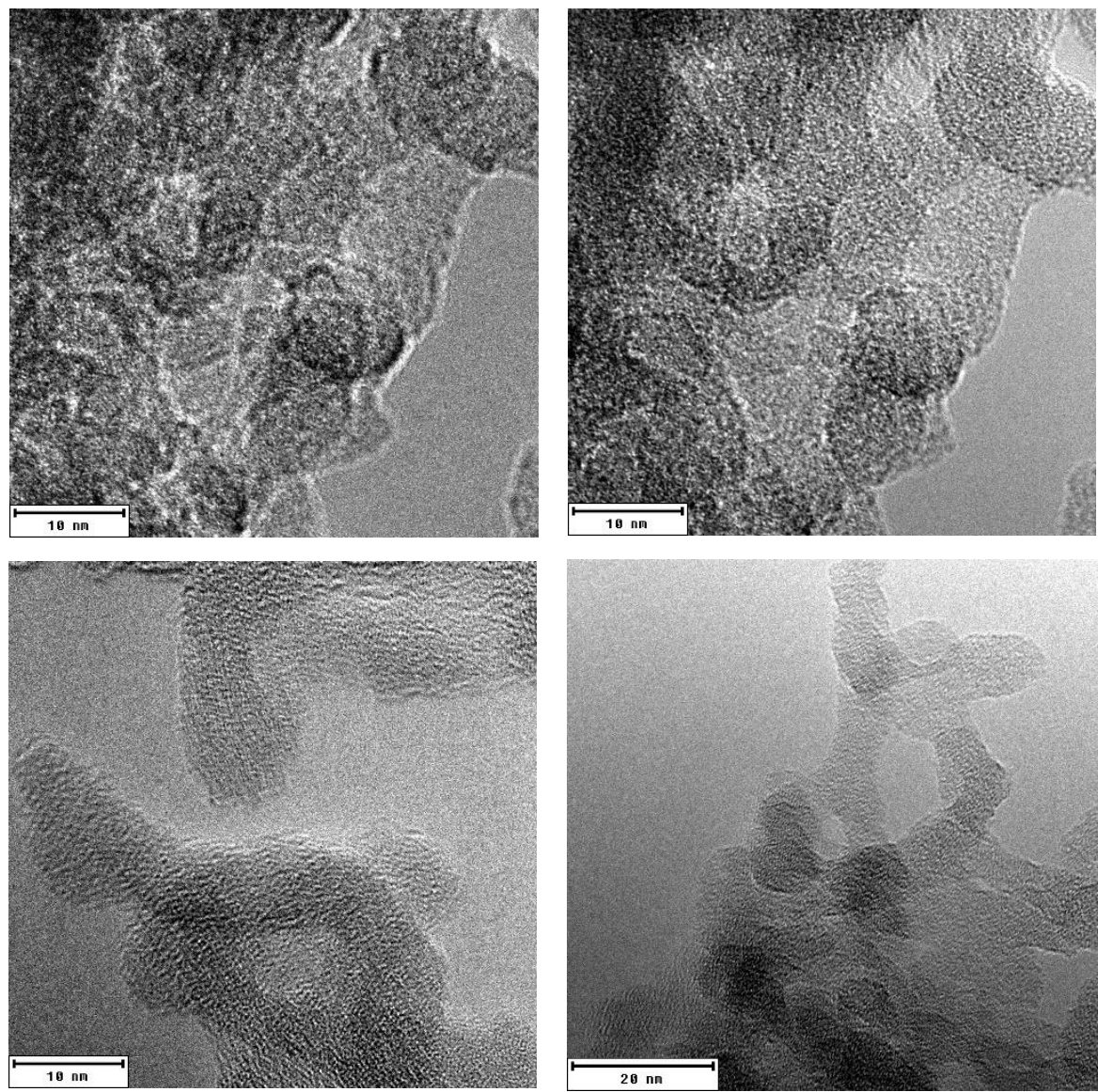

Figure S3. Microporous structure. High magnified transmission electron micrographs of SP10MTMS.

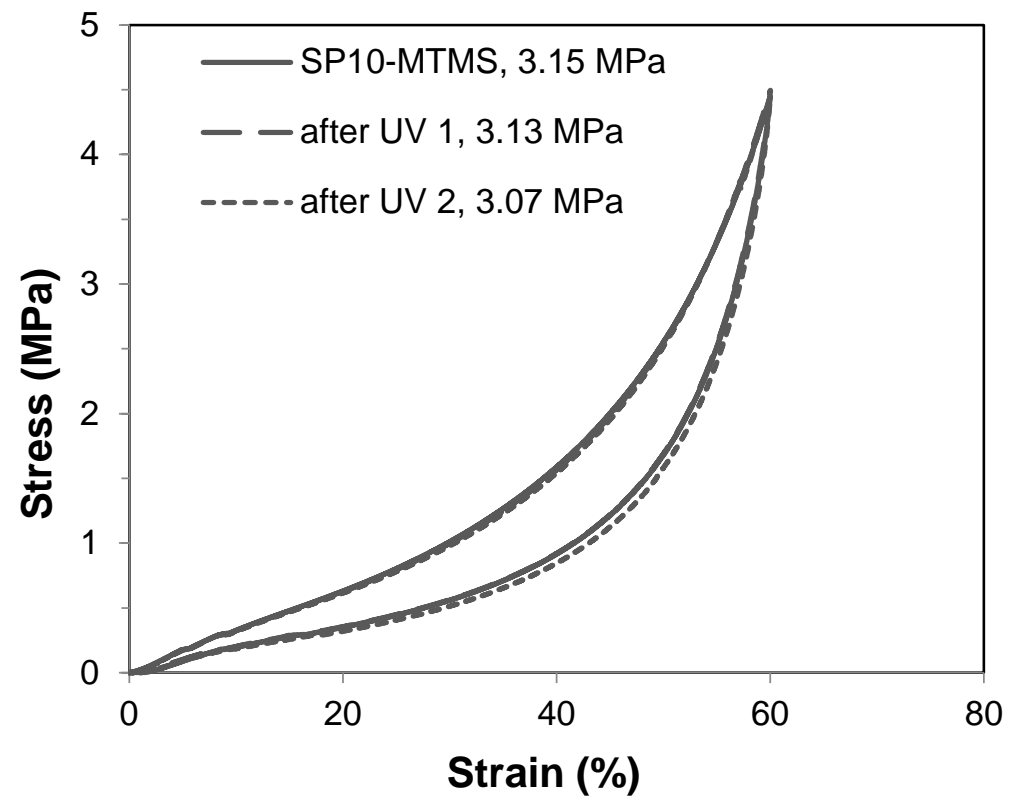

Figure S4. Stress-strain curves of compression-decompression tests for SP10-MTMS before and after the first (UV 1) and second (UV 2) conversion of the SP into the MC isomer. 


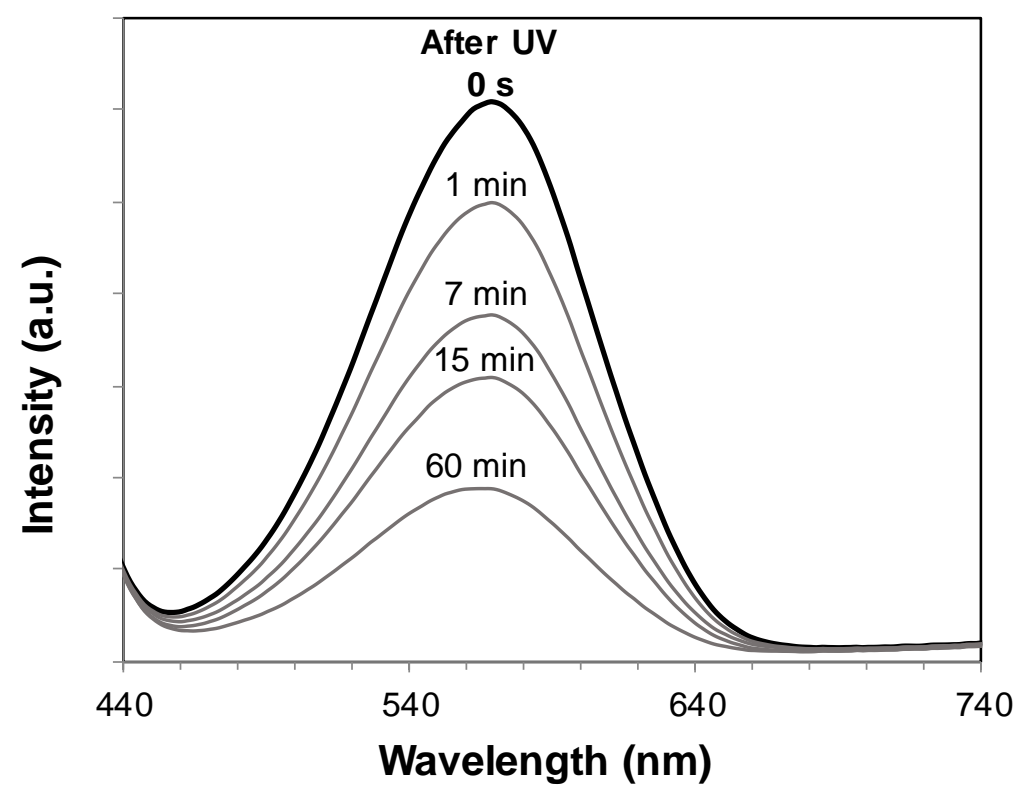

Figure S5. Kinetic investigation of SP10-MTMS in dark environment after UV irradiation for 3 min.

a)

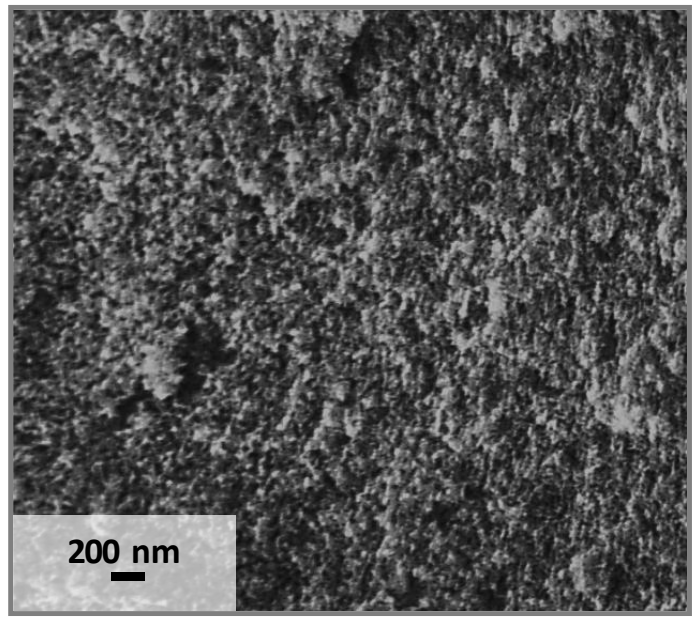

b)

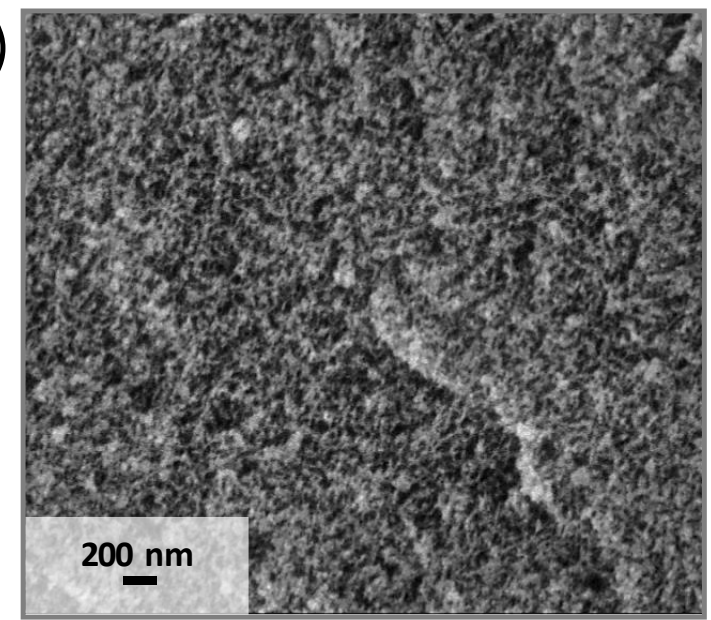

Figure S6. Scanning electron micrographs (SEM) of (a) SP0-MTMS and (b) SP10-MTMS. 